\title{
Extracellular HSP70/HSP70-PCs regulate hepatocarcinoma cell migration and invasion via RhoA
}

\author{
ZHE YI ${ }^{1}$, YAN LI $^{2,3}$, DAN LIU $^{2}$, JINGANG LIU $^{2}$ and HANGYU LI ${ }^{2}$ \\ ${ }^{1}$ School of Stomatology, China Medical University, Shenyang, Liaoning 110001; ${ }^{2}$ Department of General Surgery, \\ The Fourth Affiliated Hospital of China Medical University, Shenyang, Liaoning $110032 ;{ }^{3}$ Department of Oncology, \\ Tumor Angiogenesis and Microenvironment Laboratory (TAML), First Affiliated Hospital, \\ Liaoning Medical University, Jinzhou, Liaoning 121001, P.R. China
}

Received March 15, 2015; Accepted July 20, 2016

DOI: $10.3892 / 01.2016 .5551$

\begin{abstract}
The effects of heat shock protein 70 (HSP70)/HSP70-peptide complexes (HSP70-PCs) on the invasion and metastasis ability of hepatocellular carcinoma (HCC) Huh-7 cells were investigated. Wound healing assay revealed that cells treated with HSP70/HSP70-PCs healed faster than negative control cells. HSP70/HSP70-PCs-treated cells also exhibited better migration ability and higher invasion ability than control cells. HSP70/HSP70-PCs treatment did not alter the messenger RNA (mRNA) or protein levels of matrix metalloproteinase-9; the opposite was true for Ras homolog family member A (RhoA) mRNA and protein levels. RNA interference of RhoA attenuated the migration of HSP70/HSP70-PCs-treated cells. The present findings indicate that regulation of HCC cell migration by HSP70/HSP70-PCs occurs via regulation of RhoA expression.
\end{abstract}

\section{Introduction}

Hepatocellular carcinoma (HCC) is one of the most common malignancies worldwide, and its annual incidence approaches $\sim 0.626$ million cases worldwide (1). HCC ranks sixth among the most common malignant tumors, and is the third most common cause of cancer-associated mortality (1). Invasion and metastasis are important factors that affect the survival and quality of life of HCC patients (2). The high rates of metastasis and recurrence are major obstacles to improve the rate of survival (3).

Heat shock protein 70 (HSP70) is widely present in prokaryotic and eukaryotic cells (4). It can serve as a vehicle,

Correspondence to: Professor Hangyu Li, Department of General Surgery, The Fourth Affiliated Hospital of China Medical University, 36 Sanhao Street, Heping, Shenyang, Liaoning 110032, P.R. China

E-mail: sj_li_hangyu@sina.com

Key words: heat shock protein 70, hepatocarcinoma, RhoA, cytoskeleton acting as a 'molecular chaperone', and is capable of regulating cellular signals (5). It has been reported that numerous tumor cells release HSP70 (6). A previous study demonstrated that HSP70-peptide complexes (HSP70-PCs) released into the tumor microenvironment could not only regulate tumor immune response, but were also involved in regulating a variety of tumor biological behaviors (7). Borges et al (8) observed that extracellular HSP70 inhibited interleukin 10 expression, while Wu et al (6) noted that, in liver cancer, extracellular HSP70 enhanced resistance to apoptosis. In view of these findings, studying the impact of extracellular HSP70-PCs on tumor invasion and metastasis would be worthwhile. In the present study, the effect of extracellular HSP70/HSP70-PCs on $\mathrm{HCC}$ cell motility was investigated, and it was observed that extracellular HSP70/HSP70-PCs regulated HCC cell migration by modulating Ras homolog family member A (RhoA) expression.

\section{Materials and methods}

Cell culture. The human HCC cell line Huh-7 was purchased from the Cell Bank of the Shanghai Institute for Biological Sciences (Shanghai, China). Cells were cultured at $37^{\circ} \mathrm{C}$ in a $5 \% \mathrm{CO}_{2}$ atmosphere, in Dulbecco's modified Eagle's medium (DMEM; HyClone; GE Healthcare Life Sciences, Logan, UT, USA) supplemented with $10 \%$ fetal bovine serum (FBS; Gibco; Thermo Fisher Scientific, Inc., Waltham, MA, USA) for 2-3 days, digested with $0.25 \%$ trypsin and passaged. Logarithmic growth-phase cells were used for the experiments. Cells were divided into the following groups: Control and treated with extracellular HSP70/HSP70-PCs (final concentration, $2 \mu \mathrm{g} / \mathrm{ml})$.

Antibodies. The following antibodies were used for western blotting analyses: Rabbit monoclonal anti-HSP70 (dilution, 1:1,000; catalog no., K0414; Santa Cruz Biotechnology, Inc., Dallas, TX, USA), mouse polyclonal anti-RhoA/B/C (dilution, 1:1,000; catalog no., E2213; Santa Cruz Biotechnology, Inc.) and mouse monoclonal anti-glyceraldehyde-3-phosphate dehydrogenase (dilution, 1:2,000; catalog no., SAB2701826; GAPDH; Sigma-Aldrich, St. Louis, MO, USA). Horseradish peroxidase-conjugated sedondary antibodies were purchased 
from Beijing Zhongshan Golden Bridge Biotechnology, Co., Ltd. (Beijing, China).

HSP-peptide binding of extracellular HSP70/HSP70-PCs. In vitro binding of antigen peptide and glutathione $\mathrm{S}$-transferase (GST)-HSP70 was performed using GST-HSP70 fusion proteins (Sigma-Aldrich) as previously described (9). The reaction system contained $1 \mathrm{mmol} / \mathrm{l}$ adenosine diphosphate, $1 \mathrm{mmol} / 1 \mathrm{MgCl}_{2}, 75 \mu \mathrm{g} / \mathrm{ml}$ peptide and $250 \mu \mathrm{g} / \mathrm{ml}$ GST-HSP70, and was incubated at $37^{\circ} \mathrm{C}$ in a water bath for $2 \mathrm{~h}$. The peptides were extracted and purified from hepatocarcinoma tissue samples, as previously described (10).

Wound healing assay. A lesion was created using a plastic pipette tip $24 \mathrm{~h}$ after extracellular HSP70/HSP70-PCs treatment, and cells were washed twice with phosphate-buffered saline to remove debris. The monolayer was then maintained in serum-free DMEM and cultured for $24 \mathrm{~h}$. Then, five randomly selected fields at the border of the lesion were viewed under an inverted microscope (IX71; Olympus Corporation, Tokyo, Japan).

Transwell invasion and migration assays. Cell invasion and migration assays were performed using a Transwell system (Corning Incorporated, Corning, NY, USA) according to the manufacturer's protocol. To assess invasion ability, membranes were pre-coated with Matrigel (BD Biosciences, Franklin Lakes, NJ, USA). Approximately $5 \times 10^{4}$ cells in serum-free medium were added to the top chamber; the bottom chamber was filled with DMEM containing 10\% FBS. After 24-h incubation, cells on the upper surface of the membrane were gently removed with a cotton swab, and the membrane was then fixed in $4 \%$ methanol for $30 \mathrm{~min}$ and stained with $0.1 \%$ crystal violet for $30 \mathrm{~min}$. Cells that had migrated to the bottom surface of the membrane were collected, and the number of cells was counted. The same experimental design was used for the migration assay, but the membranes were not pre-coated with Matrigel.

Reverse transcription-quantitative polymerase chain reaction $(R T-q P C R)$. Total RNA was isolated from the cells at 24 and $48 \mathrm{~h}$ after induction with TRIzol (Invitrogen; Thermo Fisher Scientific, Inc.), according to the manufacturer's protocol. Single-strand complementary DNA was synthesized from $1 \mu \mathrm{g}$ total RNA by RT, according to the manufacturer's protocol (Toyobo Co., Ltd., Osaka, Japan). qPCR was used to measure the RhoA messenger RNA (mRNA) levels in the cells. Amplification of the cDNA was performed using the SYBR Premix Ex Taq ${ }^{\mathrm{TM}}$ II kit (Takara Bio, Inc., Otsu, Japan) qPCR was performed using an ABI PRISM ${ }^{\circledR} 7000$ Sequence Detection System (Applied Biosystems; Thermo Fisher Scientific, Inc.). Amplification was conducted in a $25-\mu 1$ volume for 35 cycles, and the product was detected using SYBR Green I fluorochrome. The cycling conditions for PCR were: $15 \mathrm{~min}$ incubation at $95^{\circ} \mathrm{C}$, followed by 45 cycles of $94^{\circ} \mathrm{C}$ for $5 \mathrm{sec}$, $50^{\circ} \mathrm{C}$ for $15 \mathrm{sec}$ and $72^{\circ} \mathrm{C}$ for $10 \mathrm{sec}$. The primers used were: RhoA forward, 5'-CCGCCATCGCTTACA-3' and reverse, 5'-GGCACCTGACCCTTGTA-3'; and GAPDH forward, 5'-GGATTTGGTCGTATTGGG-3' and reverse, 5'-TCGCTC CTGGAAGATGG-3'. GAPDH was used as a control. The $\Delta \Delta \mathrm{Cq}$ method was used for normalization (11).
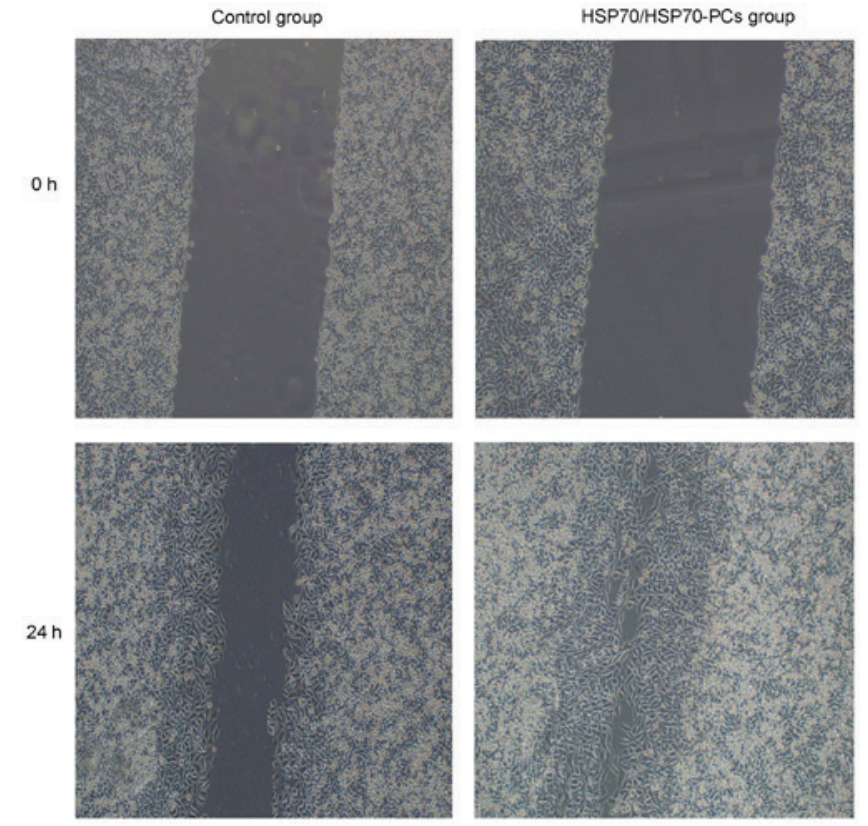

Figure 1. The effect of extracellular HSP70/HSP70-PCs on hepatocellular carcinoma cell migration. The effect of extracellular HSP70/HSP70-PCs on Huh-7 cell migration was examined by a wound healing assay. Representative digital images were obtained at 0 and $24 \mathrm{~h}$. Magnification, x10. HSP70, heat shock protein 70; PCs, peptide complexes.

Western blot analysis. Cells were lysed at the designated time points, and equal amounts of protein lysate underwent $12 \%$ sodium dodecyl sulfate-polyacrylamide gel electrophoresis. Subsequently, proteins were transferred to polyvinylidene difluoride membranes (EMD Millipore, Billerica, MA, USA) and probed with the aforementioned primary and secondary antibodies. Bound antibodies were detected with Pierce ECL Western Blotting Substrate (Thermo Fisher Scientific, Inc.). Band densities were analyzed using ImageJ software (version 1.41; National Institutes of Health, Bethesda, MA, USA).

Transient transfection of RhoA small interfering RNA (siRNA). Transfection of RhoA-siRNA was performed using Lipofectamine $^{\mathrm{TM}} 2000$ transfection reagent (Invitrogen; Thermo Fisher Scientific, Inc.) according to the manufacturer's protocol. The siRNA duplexes were purchased from Sigma-Aldrich.

Immunofluorescence staining. Cells grown on coverslips were fixed, blocked and permeabilized, as described previously (12). Cells were then stained with fluorescein isothiocyanate (FITC)-conjugated phalloidin (5 $\mu \mathrm{g} / \mathrm{ml}$; Sigma-Aldrich) for $60 \mathrm{~min}$. Actin filaments (F-actin) were visualized and photographed with a IX71 digital inverted microscope. In total, $>200$ cells from three independent experiments were analyzed for each condition. The average number of dot or fan-like protrusions around a cell was counted as an index for lamellipodia formation (12).

Statistical analysis. Data are presented as the mean \pm standard deviation. Statistical analysis was preformed using SPSS version 17.0 (SPSS, Inc., Chicago, IL, USA) Statistical 
A
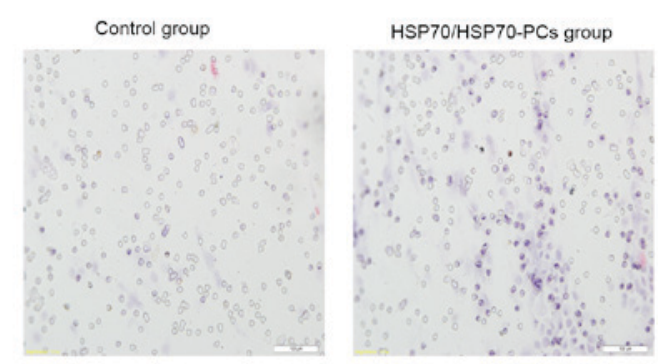

B

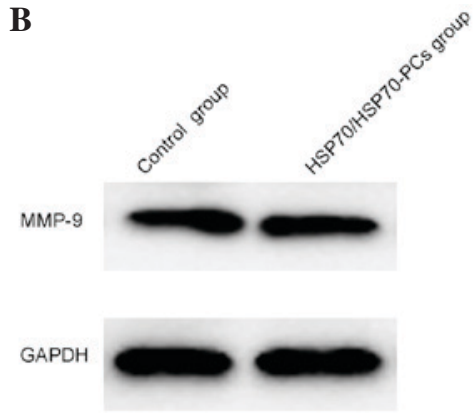

C

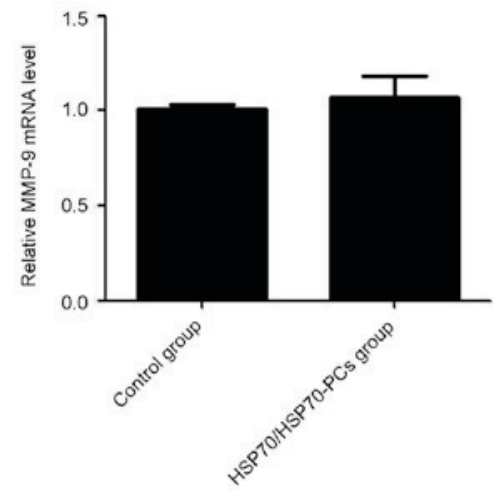

D

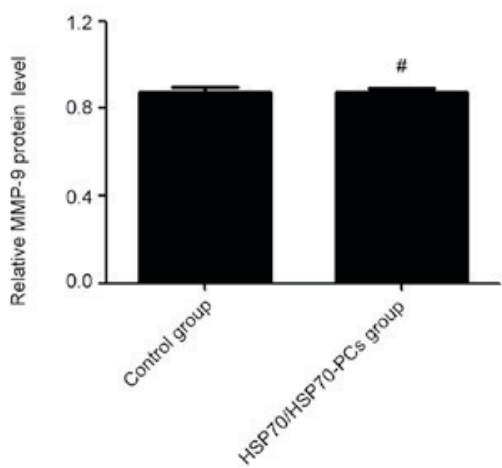

Figure 2. Extracellular HSP70/HSP70-PCs promote hepatocellular carcinoma cell invasion. (A) Effect of extracellular HSP70/HSP70-PCs on cell invasion ability, as examined by Transwell invasion assay. Magnification, x20. (B) The effect of extracellular HSP70/HSP70-PCs on the expression of MMP-9 as evaluated by western blotting. (C) The effect of extracellular HSP70/HSP70-PCs on the expression of MMP-9 as evaluated by reverse transcription-quantitative polymerase chain reaction. (D) The effect of extracellular HSP70/HSP70-PCs on the relative expression of MMP-9 by western blotting. The results are expressed as the mean \pm standard deviation from three independent experiments. ${ }^{~} \mathrm{P}<0.01$ vs. control. HSP70, heat shock protein 70; PCs, peptide complexes; mRNA, messenger RNA; MMP, matrix metalloproteinase; GAPDH, glyceraldehyde 3-phosphate dehydrogenase.

A

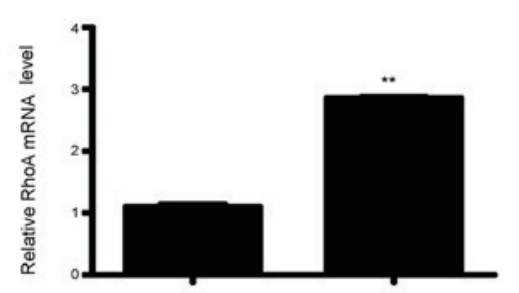<smiles>CC1CC1</smiles>

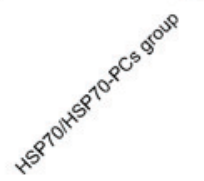

C

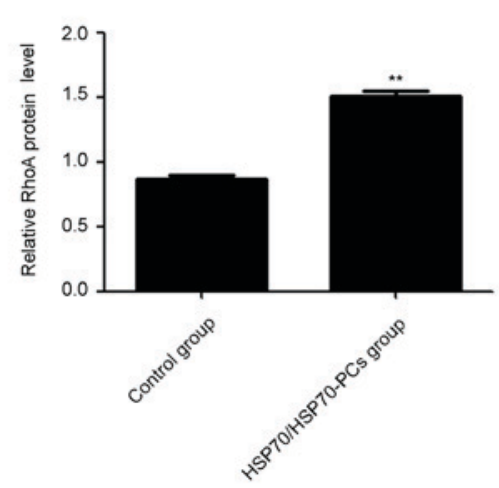

B

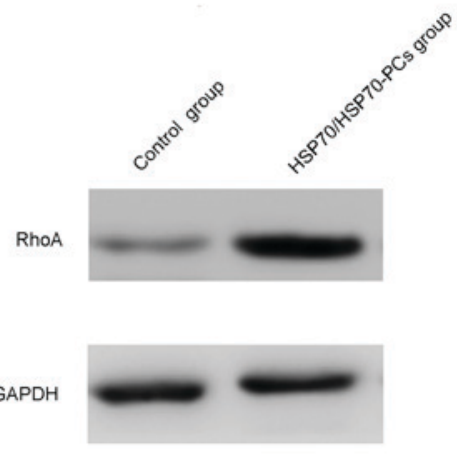

D

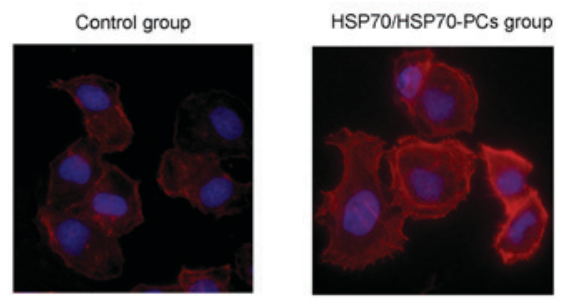

Figure 3. Extracellular HSP70/HSP70-PCs promote RhoA expression and lamellipodia formation. (A) Effect of extracellular HSP70/HSP70-PCs on the expression of RhoA, as evaluated by reverse transcription-quantitative polymerase chain reaction. (B) Effect of extracellular HSP70/HSP70-PCs on the expression of RhoA, as evaluated by western blotting. (C) The relative expression of RhoA by western blotting. (D) Lamellipodia formation was visualized by F-actin phalloidin staining upon extracellular HSP70/HSP70-PCs treatment. Cells were photographed with an Olympus IX71 digital inverted microscope. Magnification, $\mathrm{x} 40 .{ }^{* *} \mathrm{P}<0.01$. HSP70, heat shock protein 70; PCs, peptide complexes; mRNA, messenger RNA; RhoA, Ras homolog family member A; GAPDH, glyceraldehyde 3-phosphate dehydrogenase. 
significance was determined using Student's $t$-test. $\mathrm{P}<0.05$ was considered to indicate a statistically significant difference.

\section{Results}

Extracellular HSP70/HSP70-PCspromote HCC Cellmigration. To examine the role of extracellular HSP70/HSP70-PCs in $\mathrm{HCC}$ cell migration and invasion, cells were treated with extracellular HSP70/HSP70-PCs $(2 \mu \mathrm{g} / \mathrm{ml})$. HCC cell motility was then investigated using a wound healing assay. At $24 \mathrm{~h}$ after injury, the wound in the extracellular HSP70/HSP70-PCs group had healed more than that in the control group (Fig. 1).

Extracellular HSP70/HSP70-PCs promote HCC cell invasion. Migration and invasion are widely considered two closely interrelated processes (13). As extracellular HSP70/HSP70-PCs promoted HCC cell migration, the effect of extracellular HSP70/HSP70-PCs on cell invasion was next tested using Matrigel-precoated Transwell chambers. The number of invasive cells increased markedly following extracellular HSP70/HSP70-PCs treatment (Fig. 2A).

Matrix metalloproteinases (MMPs), particularly MMP-9, are crucial for cell invasion in multiple tumors, including HCC $(14,15)$. The possibility that extracellular HSP70/HSP70-PCs affect MMP-9 expression was examined in the present study. Unexpectedly, RT-qPCR and western blotting demonstrated that extracellular HSP70/HSP70-PCs altered neither mRNA nor protein levels of MMP-9 (Fig. 2B-D). Taken together, these data reveal that regulation of MMP-9 expression does not mediate the effect of extracellular HSP70/HSP70-PCs on migration and invasion.

Extracellular HSP70/HSP70-PCs promote RhoA expression. Previous studies reported that knockdown or inhibition of the small guanosine triphosphatase RhoA resulted in the inhibition of cell migration (16). Therefore, it was surmised that extracellular HSP70/HSP70-PCs may promote RhoA activation or expression, thereby inducing HCC cell elongation and/or migration. RhoA expression was measured using western blotting and RT-qPCR. Both RhoA mRNA and protein levels were significantly increased following treatment with extracellular HSP70/HSP70-PCs (Fig. 3A-C).

RhoA expression and activity are important for cytoskeletal regulation in HCC cells, including F-actin stress fiber and lamellipodia formation (17). Therefore, it was examined whether extracellular HSP70/HSP70-PCs-mediated regulation affects F-actin reorganization using FITC-conjugated phalloidin staining. Dot or fan-like protrusions were detected at the cell periphery in control cells. Following extracellular HSP70/HSP70-PCs treatment, the numbers of lamellipodia at the cell margins were markedly increased (Fig. 3D), and were consistent with our observation that cellular RhoA expression was increased upon extracellular HSP70/HSP70-PCs treatment.

RhoA is involved in extracellular HSP70/HSP70-PCsinduced HCC cell migration. To examine the possibility that RhoAisinvolvedintheeffectofextracellularHSP70/HSP70-PCs on cell migration, cells were pretreated with RhoA-siRNA, and then stimulated with extracellular HSP70/HSP70-PCs.
A

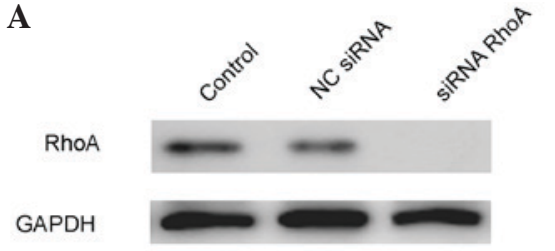

$\mathbf{B}$

Control NC SiRNA

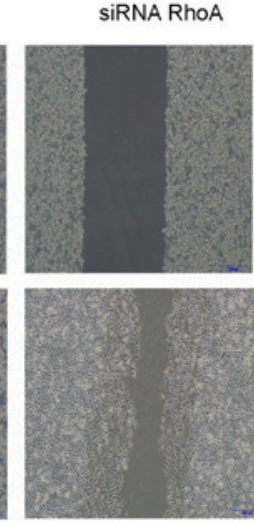

C
Control

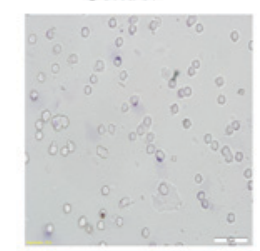

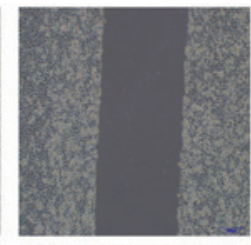

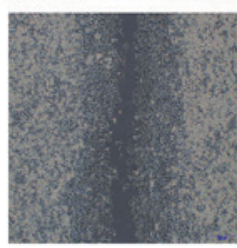

NC SiRNA

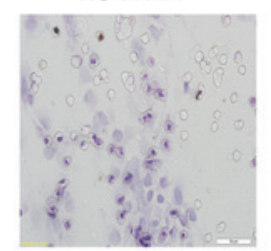

SiRNA RhoA

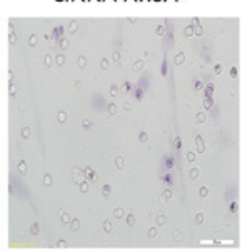

Figure 4. Extracellular HSP70/HSP70-PCs mechanism of action through RhoA. (A) Downregulation efficacy of RhoA-siRNA in Huh-7 cells. Cell lysates from NC-siRNA or RhoA-siRNA-transfected cells were subjected to western blot analysis with an anti-RhoA antibody. Glyceraldehyde 3-phosphate dehydrogenase was used as a loading control. (B) Effect of RhoA downregulation on Huh-7 cell migration, as examined by a wound healing assay. Huh-7 cells were transfected with RhoA-siRNA or NC-siRNA, and then exposed to extracellular HSP70/HSP70-PCs (2 mg/l) for the indicated time periods. Representative digital pictures were obtained at 0 and $24 \mathrm{~h}$. Magnification, x10. (C) Effect of RhoA downregulation on cell migration, as examined by a Transwell migration assay. Huh-7 cells were transfected with RhoA-siRNA or NC-siRNA prior to be exposed to extracellular HSP70/HSP70-PCs (2 mg/l). Magnification, x20. HSP70, heat shock protein 70; PCs, peptide complexes; siRNA, small interfering RNA; $\mathrm{NC}$, negative control; RhoA, Ras homolog family member A; GAPDH, glyceraldehyde 3-phosphate dehydrogenase.

RhoA-siRNA attenuated HCC cell migration (Fig 4). These data indicate that RhoA is involved in extracellular HSP70/HSP70-PCs-induced HCC cell migration.

\section{Discussion}

HSP70 is generally expressed in living organisms, and can be released into the extracellular environment in a variety of ways (18). In the tumor microenvironment, HSP70 plays a complex and contradictory role at different stages of tumor progression. Extracellular HSP70 can inhibit early-stage tumor cell growth by activating the immune response; however, it promotes late-stage tumor growth by inhibiting the immune system (6). To date, no reports in the literature state clearly that extracellular HSP70/HSP70-PCs affect tumor cell invasion. To this end, the present study treated the hepatoma cell line Huh-7 
with purified HSP70/HSP70-PCs to observe the effects of extracellular HSP70/HSP70-PCs on HCC cells. Wound healing and migration assays revealed that extracellular HSP70/HSP70-PCs enhanced the invasion and metastasis ability of Huh-7 cells.

The present study attempted to investigate the mechanism by which extracellular HSP70/HSP70-PCs promote Huh-7 cell invasion and metastasis. It is common knowledge that MMPs, particularly MMP-9, are important factors for tumor invasion and metastasis (19). Thus, the current study detected MMP-9 mRNA and protein expression with RT-qPCR and western blotting following extracellular HSP70/HSP70-PCs treatment. The results revealed that there was no significant change in MMP-9 mRNA or protein expression in extracellular HSP70/HSP70-PCs-treated cells compared with control cells. This indicates that MMP-9 is not involved in the HSP70/HSP70-PCs-mediated regulation of Huh-7 cell invasion and metastasis. Therefore, based on a literature review (20), the present study focused on RhoA in an attempt to explain the mechanism behind the HSP70/HSP70-PCs-mediated regulation of Huh-7 cell invasion and metastasis. RHOA is an important member of the RHO gene family, and is a Ras homolog (21). At present, the association between RhoA and invasion and metastasis of malignant tumors is a popular research topic (22). RhoA-mediated regulation of tumor invasion and metastasis occurs through the activation of cytoskeletal proteins that promote myosin interaction with F-actin, leading to contractility, which enhances tumor cell movement and migration ability (23). To clarify whether RhoA is involved in the extracellular HSP70/HSP70-PCs-mediated regulation of Huh-7 cells, the effect of extracellular HSP70/HSP70-PCs on RhoA expression was detected by RT-qPCR and western blotting. RhoA mRNA and protein expression were upregulated concomitantly during extracellular HSP70/HSP70-PCs-mediated promotion of tumor cell invasion and metastasis. Phalloidin staining revealed a marked increase in the number of lamellipodia at the cell margins during extracellular HSP70/HSP70-PCs-mediated promotion of Huh-7 cell invasion and metastasis. This demonstrates that the role of extracellular HSP70/HSP70-PCs in the promotion of tumor cell invasion and metastasis may be associated with RhoA and with the generation of cytoskeletal proteins. To validate the involvement of RhoA in the process of extracellular HSP70/HSP70-PCs-mediated promotion of Huh-7 cell invasion and metastasis, RhoA expression was blocked with siRNA, and it was observed that the extracellular HSP70/HSP70-PCs-mediated promotion of Huh-7 cell invasion and metastasis was significantly decreased. Phalloidin staining revealed that, once RhoA expression had been blocked, there was no significant change in the Huh-7 cell cytoskeleton following extracellular HSP70/HSP70-PCs treatment, compared with the control. This suggests that increased Huh-7 cell invasion and metastasis following extracellular HSP70/HSP70-PCs treatment occurs via upregulated RhoA expression and promotion of cytoskeleton formation.

The present results suggest that extracellular HSP70/HSP70-PCs can promote Huh-7 cell invasion and metastasis, which is achieved by upregulating RhoA expression and promoting cytoskeleton formation. The current experiments provide a novel theoretical and experimental basis for preventing and treating liver cancer in the future.

\section{Acknowledgements}

The present study was supported by the National Natural Science Foundation of China (Beijing, China; grant no. 81071955) and the Scientific Research from the Education Department of Liaoning Province (Shenyang, China; grant no. L2010634).

\section{References}

1. Jemal A, Bray F, Center MM, Ferlay J, Ward E and Forman D: Global cancer statistics. CA Cancer J Clin 61: 69-90, 2011.

2. Kawada K, Hasegawa S, Murakami T, Itatani Y, Hosogi H, Sonoshita M, Kitamura T, Fujishita T, Iwamoto M, Matsumoto T, et al: Molecular mechanisms of liver metastasis. Int J Clin Oncol 16: 464-472, 2011.

3. Gao Q, Zhao YJ, Wang XY, Qiu SJ, Shi YH, Sun J, Yi Y, Shi JY, Shi GM, Ding ZB, et al: CXCR6 upregulation contributes to a proinflammatory tumor microenvironment that drives metastasis and poor patient outcomes in hepatocellular carcinoma. Cancer Res 72: 3546-3556, 2012.

4. Mamelak D, Mylvaganam M, Whetstone H, Hartmann E, Lennarz W, Wyrick PB, Raulston J, Han H, Hoffman P and Lingwood CA: Hsp70s contain a specific sulfogalactolipid binding site. Differential aglycone influence on sulfogalactosyl ceramide binding by recombinant prokaryotic and eukaryotic hsp70 family members. Biochemistry 40: 3572-3582, 2001.

5. Schildkopf P, Frey B, Ott OJ, Rubner Y, Multhoff G, Sauer R, Fietkau R and Gaipl US: Radiation combined with hyperthermia induces HSP70-dependent maturation of dendritic cells and release of pro-inflammatory cytokines by dendritic cells and macrophages. Radiother Oncol 101: 109-115, 2011

6. Wu FH, Yuan Y, Li D, Liao SJ, Yan B, Wei JJ, Zhou YH, Zhu JH, Zhang GM and Feng ZH: Extracellular HSPA1A promotes the growth of hepatocarcinoma by augmenting tumor cell proliferation and apoptosis-resistance. Cancer Lett 317: 157-164, 2012.

7. Schmitt E, Gehrmann M, Brunet M, Multhoff G and Garrido C: Intracellular and extracellular functions of heat shock proteins: Repercussions in cancer therapy. J Leukoc Biol 81: 15-27, 2007.

8. Borges TJ, Lopes RL, Pinho NG, Machado FD, Souza AP and Bonorino C: Extracellular Hsp70 inhibits pro-inflammatory cytokine production by IL-10 driven down-regulation of C/EBP $\beta$ and C/EBPS. Int J Hyperthermia 29: 455-463, 2013.

9. Blachere NE, Li Z, Chandawarkar RY, Suto R, Jaikaria NS, Basu S, Udono H and Srivastava PK: Heat shock protein-peptide complexes, reconstituted in vitro, elicit peptide-specific cytotoxic T lymphocyte response and tumor immunity. J Exp Med 186: 1315-1322, 1997.

10. Li H, Li Y, Liu D, Sun H, Su D, Yang F and Liu J: Extracellular HSP70/HSP70-PCs promote epithelial-mesenchymal transition of hepatocarcinoma cells. PLoS One 8: e84759, 2013.

11. Livak and Schmittgen: Analysis of relative gene expression data using real-time quantitative PCR and the $2-\Delta \Delta \mathrm{Ct}$ method. Methods 25: 402-408, 2001.

12. Guo D, Sun W, Zhu L, Zhang H, Hou X, Liang J, Jiang X and Liu C: Knockdown of BDNF suppressed invasion of HepG2 and HCCLM3 cells, a mechanism associated with inactivation of RhoA or Racl and actin skeleton disorganization. APMIS 120: 469-476, 2012.

13. Wang H, Liu W, Wei D, Hu K, Wu X and Yao Y: Effect of the LPA-mediated CXCL12-CXCR4 axis in the tumor proliferation, migration and invasion of ovarian cancer cell lines. Oncol Lett 7: 1581-1585, 2014.

14. Yue P, Gao ZH, Xue X, Cui SX, Zhao CR, Yuan Y, Yin Z, Inagaki Y, Kokudo N, Tang W and Qu XJ: Des- $\gamma$-carboxyl prothrombin induces matrix metalloproteinase activity in hepatocellular carcinoma cells by involving the ERK1/2 MAPK signalling pathway. Eur J Cancer 47: 1115-1124, 2011.

15. Ghasemi R, Ghaffari SH, Momeny M,Pirouzpanah S, Yousefi M, Malehmir M, Alimoghaddam K and Ghavamzadeh A: Multitargeting and antimetastatic potentials of silibinin in human HepG-2 and PLC/PRF/5 hepatoma cells. Nutr Cancer 65: 590-599, 2013.

16. Lin Y, Rao J, Zha XL and Xu H: Angiopoietin-like 3 induces podocyte F-actin rearrangement through integrin $\alpha(\mathrm{V})$ $\beta_{3} /$ FAK/PI3K pathway-mediated rac1 activation. Biomed Res Int 2013: 135608, 2013. 
17. Le Dréan G, Haure-Mirande V, Ferrier L, Bonnet C, Hulin P, de Coppet P and Segain JP: Visceral adipose tissue and leptin increase colonic epithelial tight junction permeability via a RhoA-ROCK-dependent pathway. FASEB J 28: 1059-1070, 2014. [Epub ahead of print].

18. Mambula SS and Calderwood SK: Heat Shock Protein 70 is secreted from tumor cells by a nonclassical pathway involving lysosomal endosomes. J Immunol 177: 7849-7857, 2006.

19. van Kempen LC and Coussens LM: MMP9 potentiates pulmonary metastasis formation. Cancer Cell 2: 251-252, 2002.

20. Grise F, Bidaud A and Moreau V: Rho GTPases in hepatocellular carcinoma. Biochim Biophys Acta 1795: 137-151, 2009.
21. Strutt DI, Weber U and Mlodzik M: The role of RhoA in tissue polarity and Frizzled signalling. Nature 387: 292-295, 1997.

22. Cachero TG, Morielli AD and Peralta EG: The small GTP-binding protein RhoA regulates a delayed rectifier potassium channel. Cell 93: 1077-1085, 1998.

23. Valtcheva N, Primorac A, Jurisic G, Hollmén M and Detmar M: The orphan adhesion G protein coupled receptor GPR97 regulates migration of lymphatic endothelial cells via the small GTPases RhoA and Cdc42. J Biol C 288: 35736-35748, 2013. 